\title{
Inferred lonic Charge States for Solar Energetic Particle Events from 2012-2015 with ACE and STEREO
}

\author{
A. W. Labrador, ${ }^{1, *}$, L. S. Sollitt ${ }^{2}$, C. M. S. Cohen ${ }^{1}$, A. C. Cummings ${ }^{1}$, R. A. \\ Leske $^{1}$, G. M. Mason ${ }^{3}$, R. A. Mewaldt ${ }^{1}$, E. C. Stone ${ }^{1}$, T. T. von Rosenvinge ${ }^{4}$, \\ and M. E. Wiedenbeck ${ }^{5}$
}

${ }^{1}$ California Institute of Technology, Pasadena, CA 91125 USA

${ }^{2}$ Department of Physics, The Citadel, Charleston, SC 29409 USA

${ }^{3}$ JHU/Applied Physics Lab, Laurel, MD 20723 USA

${ }^{4}$ NASA/Goddard Space Flight Center, Greenbelt, MD 20771 USA

${ }^{5}$ Jet Propulsion Laboratory, Pasadena, CA 91109 USA

E-mail: labrador@srl.caltech.edu

\begin{abstract}
Mean ionic charge states for SEP events can reflect source temperatures, stripping during acceleration and transport, and the composition of source material. Multi-spacecraft measurements of mean ionic charge states for single SEP events can also demonstrate longitudinal dependence depending on seed particle composition or acceleration conditions. in previous studies, we calculated inferred high-energy ionic charge states for SEP events. The analysis method fits the energy dependence of decay times for each element in SEP events, combined with charge-to-mass ratios relative to a calibration element, and derives mean charge state estimates for elements from $\mathrm{O}$ to Fe. Previously, we applied the method using ACE and STEREO data to SEP events through the beginning of 2012, in order to elucidate evidence on seed populations or longitudinal variations with charge state for single SEP events, with varying results. In this paper, we continue applying the method to new SEP events from 2012 to 2015 in the ACE and STEREO data. With the three spacecraft widely spread apart during this time period, there are fewer single SEP events with multi-spacecraft data, but the wide spacing allows more SEP candidate events to be considered, separately, than would be available with just a single spacecraft. Our new results for two SEP events continue to be consistent with observed correlation between $\mathrm{Q}(\mathrm{Fe})$ and $\mathrm{Fe} / \mathrm{O}$ in previous events.
\end{abstract}

* Speaker 


\section{Introduction}

During acceleration and transport, solar energetic particles may be stripped of electrons at their origins in high temperature plasmas, or as a result of the acceleration itself, or during propagation through regions of relatively dense coronal or interplanetary material close to the sun [1-4]. As such, the mean ionic charge states of solar energetic particle events generated by flares or coronal mass ejections may indicate the temperatures of the source plasmas, or they may indicate that the SEPs were accelerated out of a seed population stripped in prior SEP events, or some combination.

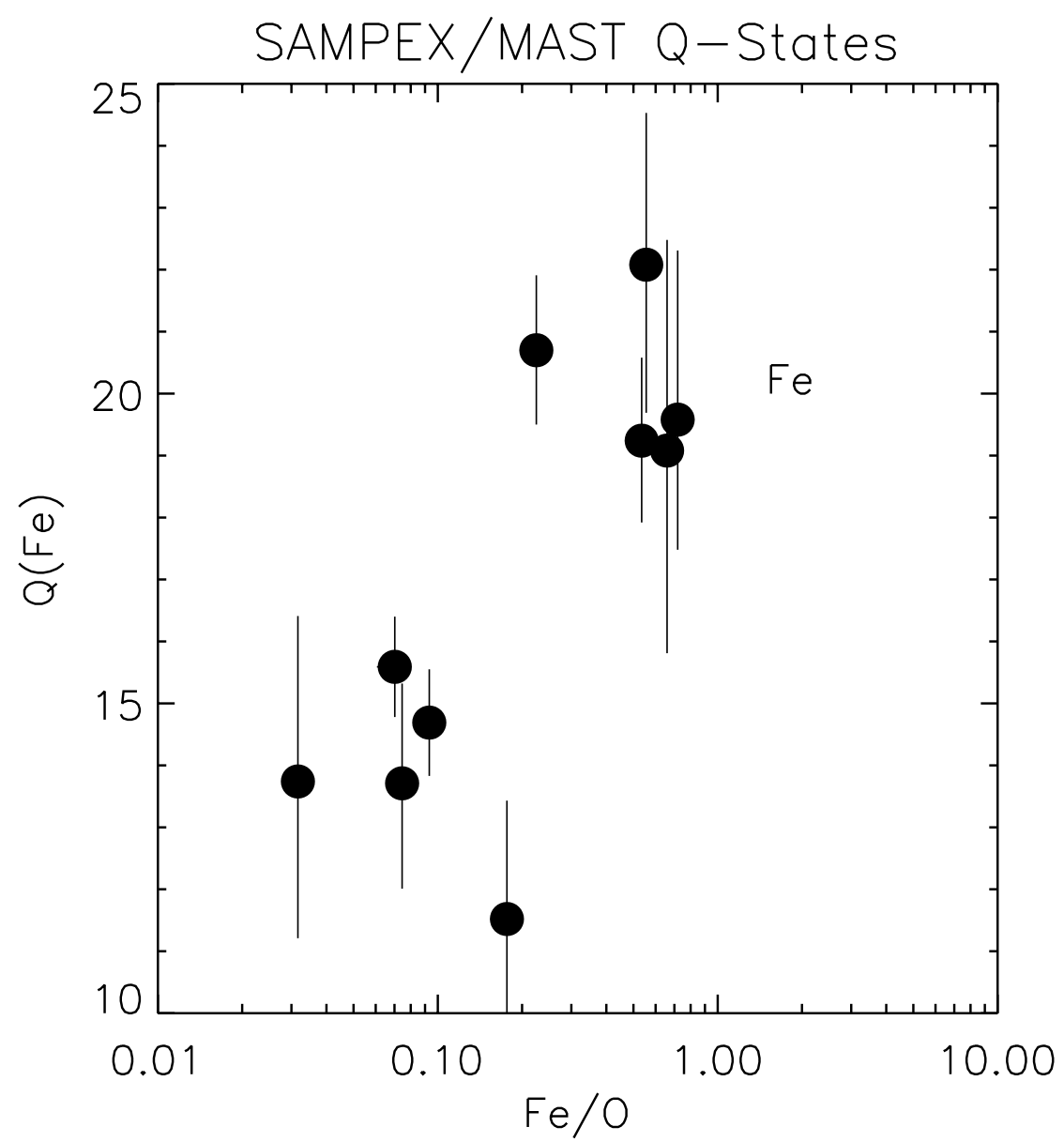

Figure 1: $Q(\mathrm{Fe})$ vs. $\mathrm{Fe} / \mathrm{O}$ as measured by SAMPEX/MAST for various large SEP events. $Q(\mathrm{Fe})$ data taken from [6].

Mean ionic charge state measurements in isolation are unlikely to determine any single cause of the ionization state, but when combined with other measurements or observations, they do provide insight. For example, mean ionic charge state measurements of SEP events by the Mass Spectrometer Telescope aboard the Solar Anomalous and Magnetospheric Particle Explorer (SAMPEX/MAST) showed correlation between mean iron charge state $(\mathrm{Q}(\mathrm{Fe}))$ for each SEP event and the iron to oxygen ratio $(\mathrm{Fe} / \mathrm{O})[5,6]$. See Figure 1. Similar correlation 
between $\mathrm{Q}(\mathrm{Fe})$ and $\mathrm{Fe} / \mathrm{O}$ ratio was observed at lower energies by the Solar Energetic Particle Ionic Charge Analyzer (SEPICA) aboard the Advanced Composition Explorer (ACE) [7]. If the cause of such a correlation were to bias in favor of mass/charge, we would expect to see an anticorrelation instead. Additionally, high $\mathrm{Q}(\mathrm{Fe})$ measurements have been observed to be more prevalent in SEP events in which the spacecraft are magnetically well connected to the solar source event.

Ionic charge state measurements at low energies (below a few $\mathrm{MeV} /$ nuc) have been provided by electrostatic deflection measurements by instruments such as the ACE/SEPICA [8], but that instrument is no longer operational. The SAMPEX/MAST instrument obtained measurements via a geomagnetic rigidity cutoff technique, but that polar-orbiting spacecraft entered the atmosphere in 2012. Ionic charge states have been inferred from the Solar Isotope Spectrometer (SIS) instrument aboard ACE via element abundance measurements and modeling of mass fractionation in SEP events [9].

In this paper, we report on ongoing analysis that infers mean ionic charge states through measurements of the energy-dependent time decay profiles of elements during certain SEP events. For this analysis, we use data from both the ACE spacecraft as well as the two STEREO spacecraft, for measurements up to several hundred $\mathrm{MeV} /$ nuc. In principle, observations by multiple spacecraft of a single SEP event will also yield longitude dependent measurements of mean ionic charge states.

\section{Inferred Ionic Charge States by Time Decay Measurements}

The method this analysis employs has been described in detail along with its derivation by Sollitt et al. [10], in conjunction with its application to ACE/SIS data. We have continued employing this method both to ACE data as well as to STEREO data [11].

The technique models SEP events as filling a magnetic containment volume in the interplanetary medium. After the injection of SEPs into the containment volume is complete, particles diffuse out of the containment volume exponentially vs. time. The escape time decay constant will be energy dependent as well, with higher energy particles escaping faster. The time decay constant has the form

$$
\frac{1}{\tau_{X}}=\frac{1}{\tau_{C}}+W\left(\alpha_{X} E\right)^{\gamma}
$$

where $\tau_{X}$ is the time decay constant for a given element $X$, measured during the decay phase of the SEP event, $\tau_{\mathrm{C}}$ is a low-energy decay time due to convection and adiabatic cooling, $\gamma$ is a constant arising from mean free path rigidity-dependence, and $\mathrm{W}$ is a normalization factor. The parameter $\alpha_{X}$ is given by

$$
\alpha_{X}=\left[\frac{Q_{C}}{A_{C}} \frac{A_{X}}{Q_{X}}\right]^{\frac{2 \gamma-1}{\gamma}}
$$

where $\mathrm{Q}$ and $\mathrm{A}$ are mean ionic charge states and mass number for elements $\mathrm{C}$ and $\mathrm{X}$, where carbon (C) is chosen as a calibration element with an assumed charge state of 5.9. (Note that in 
Equation $1, \tau_{\mathrm{C}}$ does not specifically refer to the carbon time decay constant but rather to a low energy constant. We retain this labeling for consistency with Sollitt et al. [10].)

The method begins with measurements of time decay constants during SEP events for a variety of elements (usually $\mathrm{C}, \mathrm{N}, \mathrm{O}, \mathrm{Ne}, \mathrm{Mg}, \mathrm{Si}$, and $\mathrm{Fe}$ ) over available energy ranges from either the ACE/SIS instrument or one of the STEREO/LET instruments, or both. Fits are applied to the exponential decay phase of a given SEP event, and if smooth exponentially decaying time periods are not available during the SEP event (e.g. passage of shocks distorting smoothly decaying profiles), the method is inapplicable for that event. The fits are described by Sollitt et al. [10] as maximum likelihood fits, but for this paper, high intensity events were selected for which least-squares fitting of the time decay profiles was sufficiently accurate.

With time decays, $\tau_{\mathrm{X}}$, measured for several elements and across several energy bins, the data are then fit to Equation (1). The downhill simplex method ("amoeba") is employed to vary $\mathrm{W}, \gamma, \tau_{\mathrm{C}}$, and all of the $\alpha_{\mathrm{X}}$ 's in order to find the best fit to Equation (1), by either least squares or maximum likelihood. The final, best-fit values for $\gamma$ and the $\alpha_{x}$ 's yield charge states $Q$, and constant contours of maximum likelihood or $\Delta \chi^{2}$ yield charge state uncertainties, $\sigma_{\mathrm{Q}}$.

Many factors may contribute to the inapplicability of this method to a given SEP event. First among these factors is the absence in the SEP event of a smoothly, exponentially decaying time profile for many elements for a period of sufficient duration and sufficiently high intensities for exponential decay fits to be applied with useful statistical accuracy. The second factor is an absence of the expected energy dependence of the form given in Equation (1). And the third factor is the presence of the expected energy dependence, but weakly-dependent, so that the variation of $\alpha_{x}$ 's vs. chisquare or maximum likelihood by the downhill simplex algorithm is so broad that the algorithm fails to converge to a solution, that the resulting uncertainties $\left(\sigma_{Q}\right.$ 's) are impractically large, or both. Finally, for any given SEP event, the large number of possible start and stop times for the decay profile fitting, as well as the large possible parameter space of $\mathrm{W}, \gamma, \tau_{\mathrm{C}}$, and $\alpha_{\mathrm{X}}$ 's for the downhill simplex to explore, as well as the large possible number of starting simplexes, can make fits to some SEP events more difficult.

\section{Results}

Candidate SEP events for new analysis were 5 Mar 2013 and 7 Nov 2013 for STEREO A; 13 May 2013, 25 February 2014, and 1 August 2014 for STEREO B; and 8 March 2012, 11 April 2013, 22 May 2013, and 8 January 2014 for ACE. These SEP events were selected as promising candidates for fitting the model to the data based on the abundance of Fe detected by STEREO/LET and ACE/SIS as well as the availability of exponential decay periods observed during initial event selection. Of these events, only the 8 March 2012 event readily yielded charge states and uncertainties. The failure of the method for the other events can be attributed to one or more of the factors cited above. Therefore, we have continued to explore other possible candidate SEP events not previously listed, and the SEP event of 28 September 2012 also yielded charge states in STEREO/LET A data. Here we report these new results. 

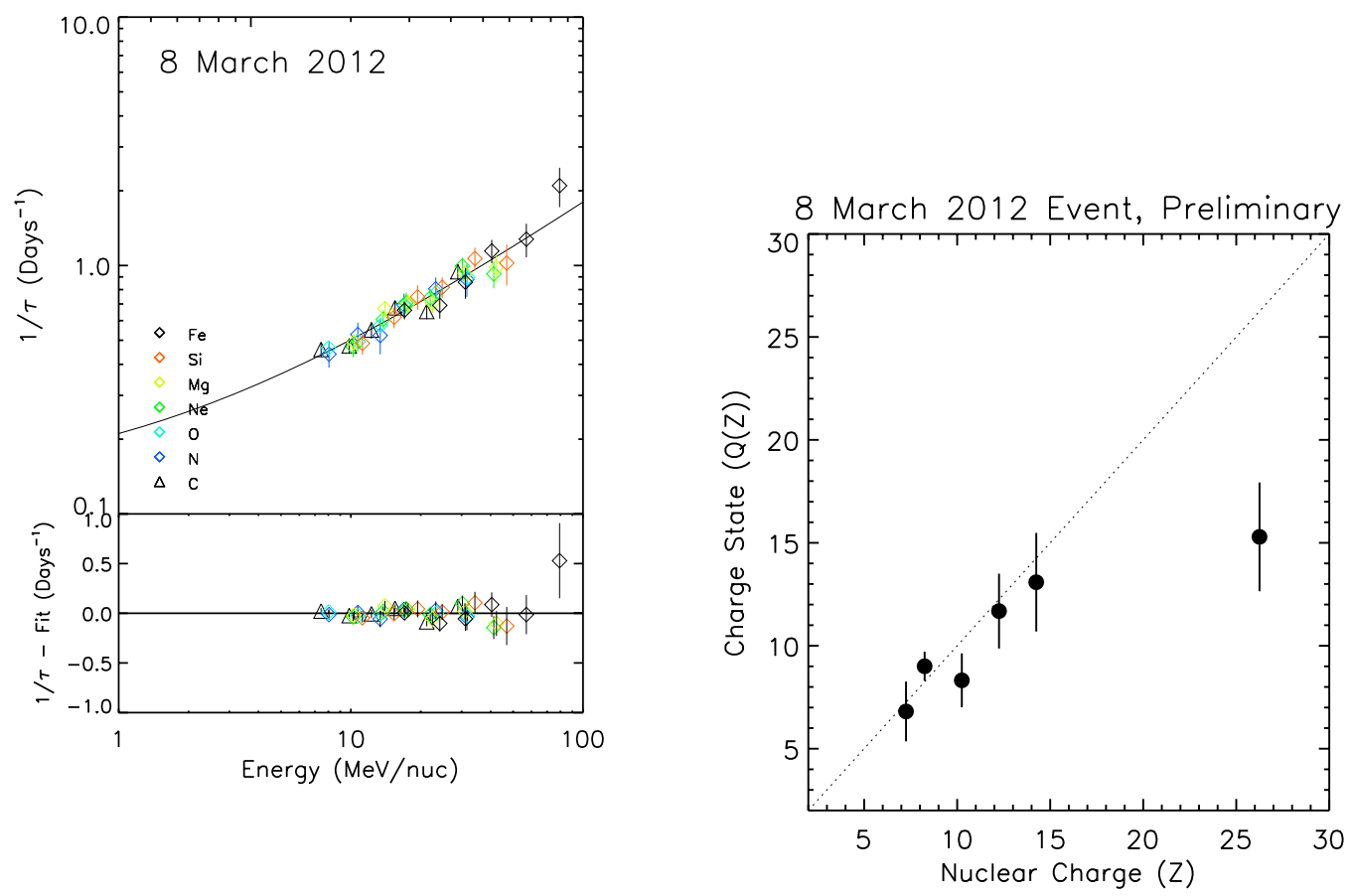

Figure 2: Final fits to Equation (1) (left) and resulting mean ionic charge states (right) for the 8 March 2012 event, with data from ACE/SIS.
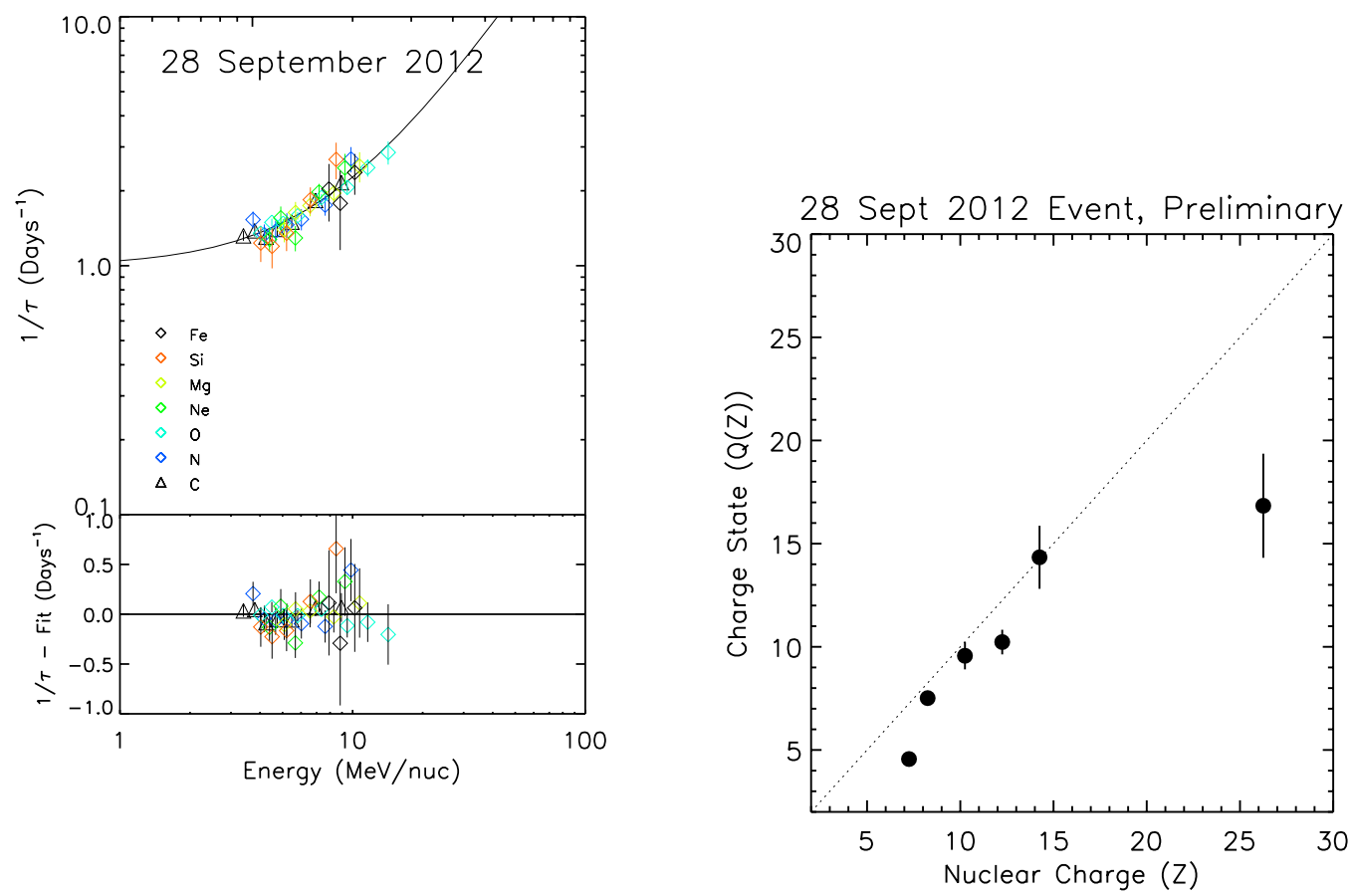

Figure 3: Final fits to Equation (1) (left) and resulting mean ionic charge states (right) for the 28 September 2012 event with data from STEREO/LET Ahead. 
Figures 2 and 3 show the results of the inferred mean ionic charge state method applied to these two events. The 8 March 2012 event in ACE/SIS data shows almost ideal behavior in the energy dependence of the time decays, with not only strong energy dependence (shown in the slope of the fit in Figure 2) but also small scatter around the fit. The 28 September 2012 event showed stronger energy dependence in the fit as demonstrated by the steeper slope at higher energies, resulting in smaller charge state uncertainties for most elements than for the earlier event, but the scatter of measured time decay constants around the final fit for $\mathrm{Si}$ and Fe resulted in relatively larger uncertainties for those elements.

These results may be compared with the high energy $\mathrm{Q}(\mathrm{Fe})$ results from SAMPEX/MAST [6] shown in Figure 1 as well as with ACE/SIS results [10]. The $Q(F e)=15.3 \pm 2.6$ value measured by ACE/SIS for the 8 March 2012 event places it in the category of low Q(Fe) events. The $\mathrm{Q}(\mathrm{Fe})=16.8 \pm 2.5$ value measured by STEREO/LET A for the 28 September 2012 event places it in the same low $\mathrm{Q}(\mathrm{Fe})$ range.

The threshold between low- and high-Fe/O ratio is usually given as the 0.134 threshold for impulsive and gradual SEP events [12]. Figure 4 shows Fe/O ratios vs. energy as measured by ACE/SIS and STEREO/LET A for the two SEP events. ACE/SIS measured Fe/O in the range 0.04 to 0.108 for $E=10.7-89.8 \mathrm{MeV} /$ nuc for the earlier event, and STEREO/LET A measured $\mathrm{Fe} / \mathrm{O}$ from 0.019 to 0.104 for $\mathrm{E}=4.5-21 \mathrm{MeV} /$ nuc. Although measured at different energies, the $\mathrm{Q}(\mathrm{Fe})$ and $\mathrm{Fe} / \mathrm{O}$ results from these two events are consistent with the previously observed correlation between $\mathrm{Q}(\mathrm{Fe})$ and $\mathrm{Fe} / \mathrm{O}$.

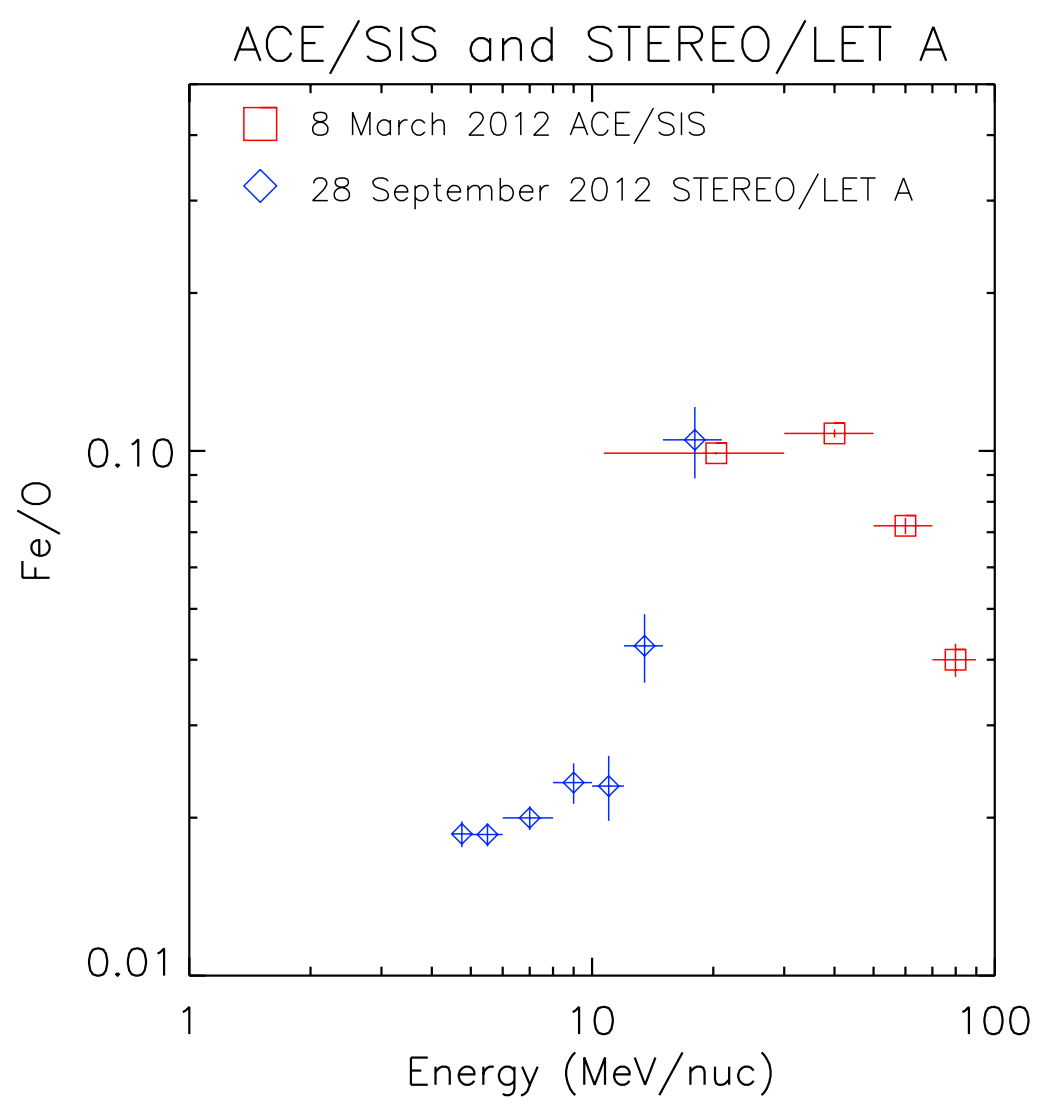

Figure 4: Fe/O ratios vs. energy as measured by ACE/SIS for the 8 March 2012 SEP event and by STEREO/LET A for the 28 September 2012 SEP event. 
The low yield of charge state results from the decay rate method points to a fuure direction for analysis: The large parameter space for start and stop times for decay time fitting as well as the very large parameter space for initial simplex for the downhill simplex method imply the need for additional automation in the search and fitting algorithm. A simple, automated method to select events and determine whether they contain smoothly exponentially decaying time periods will be more practical for surveying larger numbers of SEP events than visually scanning time profile plots. Additionally, the downhill simplex method has been somewhat unpredictable in its exploration of phase space, especially given the large number of fitting parameters. A next step is to reexamine the construction of the initial simplex and to look for methods for placing it closer to the final simplex.

\section{Acknowledgments}

This work was supported by NASA grant NNX13A66G and NASA contract NA55-03131.

\section{References}

[1] G.M. Mason et al., Solar Cycle Abundance Variations in Corotating Interaction Regions: Evidence for a Suprathermal Ion Seed Population, The Astrophysical Journal Letters 748 L31 (2012).

[2] E. Moebius et al., Strong Energy Dependence of Ionic Charge States in Impulsive Solar Events, Proc. 28th International Cosmic Ray Conf., 6, 3273 (2003).

[3] B. Klecker et al., Ionic Charge States of Solar Energetic Particles: A Clue to the Source, Space Science Reviews 130273 (2007).

[4] R.A. Mewaldt et al., Energy Spectra, Composition, and Other Properties of Ground-Level Events During Solar Cycle 23, Space Science Reviews 17197 (2012).

[5] R.A.Leske et al., Measurements of the Ionic Charge States of Solar Energetic Particles Using the Geomagnetic Field, The Astrophysical Journal Letters. 452, L149 (1995).

[6] A.W. Labrador et al., High Energy Ionic Charge State Composition in the October/November 2003 and January 20, 2005 SEP Events, Proc. 29th International Cosmic Ray Conf., Pune, 199 (2005).

[7] E. Moebius et al., Survey of Ionic Charge States of Solar Energetic Particle Events During the First Year of ACE, AIP Conf. Proceedings 528131 (2000).

[8] E Moebius et al., The Solar Energetic Particle Ionic Charge Analyzer (SEPICA) and the Data Processing Unit (S3DPU) for SWICS, SWIMS and SEPica, Space Science Reviews 86449 (1998).

[9] C.M.S. Cohen et al., New observations of heavy-ion-rich solar particle events from ACE, Geophysical Research Letters 2617 (1999).

[10] L.S. Sollitt et al., A novel technique to infer ionic charge states of solar energetic particles, The Astrophysical Journal 679910 (2008).

[11] A.W. Labrador et al., Inferred Ionic Charge States for SEPs with ACE and STEREO, Proc. 33rd International Cosmic Ray Conference, Rio de Janeiro (2013). 
Inferred Ionic Charge States for SEP Events from 2012-2015 with ACE and STEREO

[12] D.V. Reames, Energetic particles from solar flares and coronal mass ejections, AIP Conf. Proceedings 37435 (1996). 